\title{
Turismo y empleo
}

DOI: $10.22403 /$ UQROOMX/TYP02/07

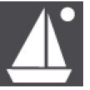

\author{
Lucinda Arroyo Arcos* \\ Edgar Manuel Gutiérrez García*
}

\section{RESUMEN}

El presente trabajo tiene como objetivo describir las variables aplicables en las investigaciones sobre el empleo turístico, con énfasis en el sector hotelero. Para su estudio se proponen dos dimensiones: una cuantitativa relacionada con número de empleos, jornada laboral, nivel educativo, nivel de ingreso, grado de calificación, población ocupada por edad, género, tipo de contratación; $y$ otra cualitativa, vinculada con: selección de personal, perfil, incentivos, capacitación, rotación, ausentismo y migración.

Palabras $\mid$ Empleo, dimensiones, turismo, variables cuantitativas y CLAVE cualitativas.

*Universidad de Quintana Roo / larroyo@uqroo.mx

**Auxiliar de Investigación 


\section{Introducción}

Debido a que la actividad turística propicia la generación de empleo a partir de la operación de empresas que procuran satisfacer las necesidades de los turistas, diversos países la han tomado como prioritaria. El hombre ha trabajado en distintas actividades por necesidad pero también para satisfacer su instinto lúdico, que es muy poderoso (Gurría 199I: 21); por lo tanto, el trabajo se realiza para obtener recursos económicos que permiten a los individuos adquirir los satisfactores que cubren sus necesidades físicas y espirituales. Así, el empleo se entiende cuando una persona, sea física o moral, paga a otra por realizar una o varias funciones en beneficio del empleador, sea con o sin contrato, de forma temporal o permanente. Se encuentran diversas acepciones de lo que es o debiera ser el empleo, para Quijano (2000):"es toda actividad humana libre, ya sea material o intelectual, que una persona ejecuta permanente y conscientemente al servicio de otra."

Al respecto, un elemento importante para explicar la generación de empleo en México es la evolución en el ámbito sectorial, que se inicia con el proceso de apertura económica y entra en una etapa de terciarización, es decir, que la participación del empleo industrial desciende, mientras la del comercio y servicios aumenta (Camargo, 1999: 27). Por consiguiente, durante los años noventa, se mantuvo una tendencia de reducción del empleo en el sector primario y secundario, y registró mayor dinamismo en el terciario. En este último reglón se destaca la rama de servicios financieros: seguros, servicios a empresas, bienes raíces y servicios básicos. Por el otro, la rama de comercio: grandes tiendas, supermercados, comercio exterior, las relativas al turismo, como restaurantes, hoteles y el comercio informal típico (Weller, 2000: 37). Lo anterior se presenta debido a la creciente competitividad de las empresas y por el interés de abarcar diversos segmentos.Así, tenemos los segmentos formados en su mayoría por turistas, extranjeros y nacionales, que reciben servicios de hospedaje, alimentación, transporte y operadores turísticos; $y$ el segmento compuesto por residentes o turistas que requieren de cafeterías, discotecas, centros recreativos, entre otros.

Por ello, la generación de empleo turístico es considerada como un beneficio que, si bien no es de lo mejor remunerado, en tiempos de rescisión es preferible tener uno de estos empleos que carecer de él (Aguilar, 1994: 10). 
Para el año 2000 el sector turístico en México se destacó como una de las principales fuentes generadoras de empleo al registar I 848814 ocupaciones, de las cuales $74.1 \%$ correspondió a empresas hoteleras. En el mismo año, el Producto Interno Bruto (PIB) por turismo, representó $8.3 \%$ del total nacional (Sectur, 2002).

A continuación, se realiza una revisión de datos estadísticos para identificar la tendencia del empleo turístico, tanto en el estado de Quintana Roo como en el municipio de Cozumel.

Para 1990, se calculó que en Quintana Roo, 90\% del PIB fue generado por las actividades turísticas, conexas y colaterales (César y Navarro, 1992: 13). Asimismo, el rubro de servicios de hoteles y restaurantes se ubicó en primer lugar con $14.5 \%$ como generador de empleos; en segundo lugar, el comercio con 13.7\%, y el tercer puesto fue ocupado por los servicios personales y de mantenimiento con 9\% (INEGI, 1990). Para el año 2000 se repite el mismo esquema, los servicios de hoteles y restaurantes ocupan el primer lugar con 18\%; en segundo término otros servicios, excepto gobierno, con $9 \%$, y en tercer lugar las actividades de gobierno, que representaron $5 \%$ (INEGI, 2000).

Respecto al municipio de Cozumel, éste basa su economía en actividades estrechamente vinculadas con el turismo y el comercio; destaca la hotelería, gastronomía y la comercialización de productos artesanales, en virtud de que generan la mayor parte de los empleos (Martínez y Collantes, 2003: 72). De acuerdo con datos estadísticos (INEGI, 1990), el rubro de servicios de hoteles y restaurantes alcanzó $21 \%$ como generador de empleos, seguido del comercio con 16\%; en tercer orden, los servicios personales y de mantenimiento con II\%. Para el año 2000, el rubro de servicios de hoteles y restaurantes se mantiene con $21 \%$; seguido de otros servicios, excepto gobierno, con $9 \%$; y en tercer orden, las actividades gubernamentales, con 4.5\% (INEGI, 2000).

En resumen, para 1990 en el estado existía tan sólo $2 \%$ de desempleo, y para el año 2000 este índice se reduce hasta 0.5\%, es decir, en Quintana Roo y Cozumel, el rubro de servicios restaurantes y hoteles mantiene una constante, ocupa el primer lugar como generador de empleos, favoreciendo al sector económico del estado y de la región.

En ese contexto, es esencial avanzar en un análisis del empleo en el sector hotelero de cada destino turístico, para tener información particular sobre sus características o dimensiones cuantitativas y cualitativas. 


\section{Metodología}

Se consultaron estudios de la CEPAL en Weller (2000), de la Secretaría de Turismo (Sectur, 1994 y 2002), las base de datos del INEGI (1990 y 2000), el Consejo de Normalización y Certificación de Competencia Laboral, Conocer (2000). Asimismo, sirvió de consulta el trabajo de tesis Caracterización del empleo en las empresas hoteleras de Cozumel (Gutiérrez, Arroyo y Santander, 2005).

Además, se llevó a cabo una revisión bibliográfica de autores expertos en el tema del empleo turístico, tales como: Shaw y Williams (1994), Vellas (2002), Aguilar (1994), Bote (1991), Hernández (1999), López (1997), y Camargo (1999), con el objeto de clarificar cada una de las dimensiones propuestas.

\section{Desarrollo}

\section{Dimensión cuantitativa}

Para orientar a los interesados en el estudio del empleo, en las siguientes líneas se describen las variables cuantitativas para la caracterización y análisis de la mano de obra del sector hotelero, entre las que se sugieren: número de empleos, jornada laboral, nivel educativo, nivel de ingreso, grado de calificación, población ocupada por edad, género, tipo de contratación, entre otros.

Número de empleos. Al respecto, el indicador establecido por la Secretaría de Turismo señala que por cada cuarto de hotel se genera un empleo directo $y$ cuatro indirectos (Botello, 2003). El empleo directo se define como el trabajo generado en las empresas que atienden o brindan un servicio de primera línea al turista, como: hoteles, agencias de viajes, restaurantes, servicios de transporte. Los tipos de empresas antes mencionadas requieren de bienes y servicios para su funcionamiento como: proveedores de insumos, energéticos, lavandería, entre otros; estos negocios crean empleos denominados indirectos. Con esta variable se busca verificar en qué medida se cumple con el indicador establecido por el organismo oficial o, por el contrario, realizar el análisis respecto de las diferencias identificadas.

Jornada laboral. El horario de trabajo se refiere al tiempo comprendido entre la hora de entrada y salida en una jornada de trabajo. En la hotelería existe 
una gran variedad y diferencia de horarios, completamente distinta al resto de la industria. Incluso los horarios pueden no ser regulares, ya que generalmente se cambian semanal o quincenalmente. El turno de trabajo que se realiza dentro de la jornada laboral puede ser variado; los hay de mañana, tarde y noche, pero también puede estar la jornada partida, o sea, dos turnos de trabajo (López, 1996: 19-20). Asimismo, el hecho de trabajar en horarios diferentes a los ordinarios, es decir, en fines de semana, fiestas, vacaciones fuera de la temporada turística, provoca dificultades de contratación, especialmente entre el personal femenino que tiene cargas de familia (Vellas, 2002: 149). Aquí, se busca identificar el promedio de horas trabajadas en el sector, según la media nacional y el esquema de las jornadas.

Nivel de educación y grado de calificación. Un estereotipo común es que el empleado del turismo no tiene educación, motivación, entrenamiento, habilidades y es improductivo (Shaw y Williams, 1994: 165). La otra postura es que la formación profesional y la especialización repercuten directamente en la productividad de las empresas y en el crecimiento de la industria turística (Sancho, 2002: 145). Por ello, este indicador es relevante dentro del análisis cuantitativo, ya que indicará cuál es el grado de educación y calificación de la mano de obra del sector y qué tipos de puestos se encuentran ocupandos.

Nivel de ingreso. El empleo turístico en México ha sido en promedio de bajas remuneraciones y ha ocupado a personal poco calificado (Sectur, 1994: 10). El pago de bajos salarios para las empresas que se instalan en países en vías de desarrollo significa una ventaja competitiva en los esquemas de competencia internacional (Chavarín, 1999:28). Según Shaw y Williams (1994:30) es necesario conocer la calidad de los empleos que se generan, porque una gran mayoría pueden ser puestos de la menor calificación con remuneraciones salariales de las más bajas. El ingreso es un indicador que permitirá conocer el tipo de percepciones que reciben los trabajadores del sector y realizar el análisis respecto de la información manejada por las fuentes oficiales.

Temporalidad y tipo de contratación. Uno de los cuestionamientos más comunes es la temporalidad que se maneja en el sector turístico. Bote (I99I) señala que la actividad turística, por ser altamente estacional, tiene entre sus consecuencias la generación de empleos inestables (contratos eventuales) y con la llegada de la temporada baja, el despido masivo de trabajadores por la caída 
de la afluencia de turistas. Ante estas afirmaciones es necesario identificar ¿qué pasa en el sector hotelero?, ¿cuál es el porcentaje de los empleos seguros e inestables?, ¿cuál es el grado de vulnerabilidad de los empleados?, con la finalidad de reflejar las características endógenas de los empleos generados en las empresas de hospedaje y buscar alternativas de atención.

\section{Dimensión cualitativa}

Aunque resulta difícil tener conocimiento sobre las variables cualitativas, se convierten en puntos importantes para la toma de decisiones en la administración de las empresas del sector hotelero. Por ello, se ponen a consideración las siguientes: selección de personal, perfil, incentivos, capacitación, rotación, ausentismo y migración.

Selección de personal. Este proceso puede entenderse como el conjunto de entrevistas, exámenes, comprobaciones, referencias y documentación, tendientes a encontrar al mejor candidato para ocupar una posición en la empresa (Schulz, 200I). Sin embargo, las prácticas en el sector hotelero pueden no respetar estos lineamientos, sujetos a la oferta y la demanda de puestos. Por ello, la importancia de corroborar qué perfil se requiere para ocupar un puesto en los distintos niveles de la organización; verificar si se siguen procesos o criterios específicos e identificar la capacidad de espacio para puestos ocupados por extranjeros.

Incentivos. Existen prestaciones establecidas de acuerdo con la Ley Federal del Trabajo; sin embargo, se intenta identificar aquellas políticas o estrategias establecidas para motivar o incentivar a los empleados que coadyuven a mejorar el ambiente laboral y hacer más eficiente su desempeño diario.

Capacitación. En México, la capacitación no cuenta con una entidad de formación oficial, así que las empresas están obligadas por ley a capacitar a su personal; pero en la mayoría de las pequeñas y medianas empresas (PYMES) esto no se respeta y los trabajadores no cuentan con el entrenamiento y conocimiento para efectuar con calidad y eficiencia sus labores (López, 1997). Como declara Hernández (1999: 176), la práctica de la capacitación en las empresas no es generalizada, concentrándose preferentemente en las empresas de mayor tamaño relativo. Si las empresas turísticas reconocieran que el trabajador es el eje fundamental y la razón de ser de la organización, se 
respetaría la capacitación, pero sólo algunas empresas visionarias concentran su atención en el desarrollo de su talento humano (Valencia, 2002:45). Como expresa Monfort (2002: 7), la formación de los trabajadores y la remuneración se valoran como elementos esenciales para la competitividad de una empresa. En ese sentido, lo interesante es indagar qué sucede en las empresas hoteleras y cuál es la oportunidad de capacitación que brindan a sus empleados.

Rotación. Ésta señala la movilidad que tiene la empresa en sus diferentes áreas y puestos. Se presenta cuando por renuncia, despido o abandono de trabajo, se genera una vacante. Esto conlleva siempre a costos que afectan a la economía de la organización, como el gasto que se realiza para el reclutamiento (anuncios, llamadas telefónicas, papelería), así como de selección (el sueldo proporcional de las personas que entrevistan, papelería, entre otros). Además, el periodo de inducción representan gastos escondidos por falta de productividad ante el desconocimiento de la empresa y servicios que ofrece, fallas o mermas, que repercuten en una mala atención a los huéspedes (Schulz, 2003). Esta variable es de suma importancia para reconocer los cuellos de botella e identificar los nichos de oportunidad que tiendan a la reducción de los costos por esta situación en las empresas del sector.

Ausentismo. Resulta de faltas injustificadas, enfermedades, accidentes de trabajo, maternidad, problemas de alcoholismo, etc. Indudablemente, si existe ausentismo se daña la cadena de servicio y la atención a los clientes. Por tanto, es recomendable que esta variable se estudie por área o departamento con la finalidad de identificar particularidades y obtener información que coadyuve a la toma de decisiones.

Migración. El empleo ha seguido las pautas de un modelo turístico de enclave en los nuevos desarrollos turísticos, es decir, una actividad económica que se implanta en una región que era tierra virgen, en la que no existe una cadena de proveedores y cuyo recurso aprovechable son las personas que ya habitan en la zona, en caso de no ser un lugar poblado, genera los flujos migratorios para contar con la fuerza de trabajo necesaria (Sectur, 1994: 10). En síntesis, si la demanda de trabajo excede el abastecimiento disponible en un distrito de mercado en particular, se requiere migración que podría ser temporal o permanente, dependiendo de la naturaleza de la demanda (Shaw y Williams, 1994: 183), de ahí el interés de reconocer el porcentaje de trabajadores del sector, procedentes de la región, estados o de otros países. 


\section{Conclusión}

En el presente trabajo se expusieron datos estadísticos avalados por la Secretaría de Turismo (Sectur) y el Instituto Nacional de Estadística, Geografía e Informática (INEGI), donde se observa que las actividades turísticas han hecho posible la generación de empleos, destacando que el sector hotelero es considerado como una de las principales fuentes de trabajo. Sin embargo, esta contribución no debe "sobrevalorarse", tampoco entenderse en términos de crecimiento económico, sino en el sentido de mejoramiento del nivel de vida de los individuos, crecimiento cultural, educativo y científico-tecnológico, niveles bajos de inseguridad y menos marginación. Para los interesados en la temática del empleo se pone a consideración la descripción de las posibles variables de estudio, por un lado las cuantitativas (nivel de ingreso, jornada laboral, nivel de educación, tipo de contrato, edad, género) y por el otro, las cualitativas (selección de personal, perfil, incentivos, capacitación, rotación, ausentismo y migración) con la finalidad de avanzar en el análisis sobre dichas características en pro de reflejar las condiciones reales en las que están inmersos los empleados del sector hotelero.

\section{FUENTES CONSULTADAS}

Aguilar,A. (1994). “Ingreso y mercado laboral en ciudades turísticas” Ciudades, Puebla: Red Nacional de Investigación Urbana (RNIU), núm. 23, 0-I8.

Bote, G.V. (I99I). Planificación económica del turismo: de una estrategia masiva a una artesanal. México:Trillas.

Botello, L. (2003) “Guía de planeación. Lo nuevo bajo el sol: destinos calientes” en Expansión, enero-febrero, México.

Camargo,J. (1999). “Apertura económica, productividad y mercado de trabajo en Argentina, Brasil y México” en V.Tokman (coord.), Productividad y empleo en la apertura económica. Lima, Perú: Oficina Internacional del Trabajo.

César, A. y D. Navarro (1992). Quintana Roo: los retos del fin de siglo. México: Centro de Investigaciones de Quintana Roo (ClQRO).

Conocer (2000). Análisis económico y laboral del sector turístico en México. México: Consejo de Normalización y Certificación de Competencia Laboral/Limusa. 
Chavarín, R. (1999). Mercados regionales de trabajo y empresa. México: Universidad de Guadalajara.

Gurría, D. M. (|99|). Introducción al turismo. México:Trillas.

Gutiérrez, E., L. Arroyo y L. Santander (2005). Caracterización del empleo en las empresas hoteleras de Cozumel, Quintana Roo. Tesis de licenciatura, Universidad de Quintana Roo, Unidad Académica Cozumel.

Hernández, L. E. (1999). "Apertura comercial, productividad, empleo y contratos de trabajo en México" en V. Tokman (coord.), Productividad y empleo en la apertura económica. Lima, Perú: Oficina Internacional del Trabajo.

INEGI (2000). Anuario estadístico, división de actividades económicas.Aguascalientes, Ags.: Instituto Nacional de Estadística, Geografía e Informática.

(1990). Anuario estadístico, división de actividades económicas. Aguascalientes, Ags.: Instituto Nacional de Estadística, Geografía e Informática.

López, C.A. ( 1996). Hostelería: curso completo de servicios: hoteles, restaurantes, cafeterías y bares. España: Paraninfo.

López G. J. (1997). Macroeconomía del empleo y políticas de pleno empleo para México. México: Porrúa.

Martínez, C.yA. Collantes (2003). Turismo en áreas rurales de Cozumel. México: Universidad de Quintana Roo.

Monfort,V.(2002).“Estrategia competitiva y desempeño en la industria hotelera costera: evidencias empíricas en Benidorm y Peñíscola” Cuadernos de Turismo, 10. España.

Quijano, P.A. (2000). Glosario de Salud ocupacional. Colombia: Universidad del Rosario.

Sancho, P. A. (2002). "Análisis de los planes de formación de las empresas del sector turístico" en Recursos humanos en turismo: un nuevo paradigma. España: Organización Mundial de Turismo.

Sectur (1994). Modernización del empleo turístico en México. Cuaderno 9, abril, México: Unidad de Política Turística, Secretaría de Turismo.

(2002). El empleo en el sector turístico. México: Secretaría de Turismo.

Shaw, G. y A.Williams (1994). Critical Issues in Tourism: a Geographical Perspective. Londres: Blackwell Publishers

Schulz, J.A. (200I). "La correcta selección de personal: Una práctica olvidada" en Alta Hotelería, septiembre-octubre, 63, 42-43. 
Schulz, J. A. (2003). "Importancia de los indicadores en el área de recursos humanos" en Alta Hotelería, enero-febrero, 66, 37-39.

I46 Valencia, M. (2002). El recurso humano:clave para el desarrollo turístico en Recursos humanos en turismo: un nuevo paradigma. España: Organización Mundial de Turismo.

Vellas, F. (2002). Economía y política del turismo internacional. España: Síntesis.

Weller, J. (2000). "Tendencias del empleo en los años noventa en América Latina y el Caribe" Revista de la CEPAL, 72, Santiago de Chile. 